\title{
Elusive electron-phonon coupling in quantitative analyses of the spectral function
}

\author{
C. N. Veenstra, ${ }^{1,2}$ G. L. Goodvin, ${ }^{1}$ M. Berciu, ${ }^{1}$ and A. Damascelli ${ }^{1,2, *}$ \\ ${ }^{1}$ Department of Physics \& Astronomy, University of British Columbia, Vancouver, British Columbia V6T 1Z1, Canada \\ ${ }_{2}^{2}$ AMPEL, University of British Columbia, Vancouver, British Columbia V6T 1Z4, Canada
}

(Dated: May 25, 2022)

\begin{abstract}
We examine multiple techniques for extracting information from angle-resolved photoemission spectroscopy (ARPES) data, and test them against simulated spectral functions for electron-phonon coupling. We find that, in the low-coupling regime, it is possible to extract self-energy and bareband parameters through a self-consistent Kramers-Kronig bare-band fitting routine and verify the momentum independence of the self-energy along the quasiparticle dispersion. We also show that the effective coupling parameters deduced from the renormalization of quasiparticle mass, velocity, and spectral weight are momentum dependent and, in general, distinct from the true microscopic coupling; the latter is thus not readily accessible in the quasiparticle dispersion revealed by ARPES.
\end{abstract}

PACS numbers: 71.38.-k, 79.60.-i, 74.25.Jb

Angle-resolved photoemission spectroscopy (ARPES) is a tool which, experimental difficulties aside, provides access to the electron-removal part of the momentumresolved spectral function $A(\mathbf{k}, \omega)$ 1]. This quantity is an extremely rich source of information since it depends on both the single-particle electronic dispersion $\varepsilon_{\mathbf{k}}^{b}$ (the socalled 'bare-band') as well as the quasiparticle self-energy $\Sigma(\mathbf{k}, \omega)=\Sigma^{\prime}(\mathbf{k}, \omega)+i \Sigma^{\prime \prime}(\mathbf{k}, \omega)$, whose real and imaginary parts account for the energy renormalization and lifetime of an electron in a many-body system. The single-particle spectral function is generally written in the form:

$$
A(\mathbf{k}, \omega)=-\frac{1}{\pi} \frac{\Sigma^{\prime \prime}(\mathbf{k}, \omega)}{\left[\omega-\varepsilon_{\mathbf{k}}^{b}-\Sigma^{\prime}(\mathbf{k}, \omega)\right]^{2}+\left[\Sigma^{\prime \prime}(\mathbf{k}, \omega)\right]^{2}} .
$$

In the case of $\mathbf{k}$-independent self-energies, methods based on the Lorentzian fit of momentum distribution curves [MDCs, i.e. constant energy cuts of $A(\mathbf{k}, \omega)]$ have been used to extract $\Sigma^{\prime}(\omega)$ and $\Sigma^{\prime \prime}(\omega)$ from the spectral function measured by ARPES, and eventually to infer information on the nature and strength of the interactions dressing the quasiparticles in a variety of complex systems [1-6]. However, the question of the validity of these approaches is still a pressing one because most methods hinge on some assumption and/or approximation for the bare-band $\varepsilon_{\mathbf{k}}^{b}$. More fundamental, for instance in the case of electron-boson coupling, the commonly assumed link between coupling strength and quasiparticle renormalization, through the so-called mass enhancement factor $(1+\lambda)$, is at best merely phenomenological and its general validity needs verification. This calls for a methodological study based on a well-behaved and momentumindependent model self-energy.

Here we investigate the possibility of extracting momentum-independent self-energies from $A(\mathbf{k}, \omega)$, without any a priori knowledge of the bare-band $\varepsilon_{\mathbf{k}}^{b}$. We will test the performance of our approach on the spectral function generated with the high-order momentumaverage approximation $\mathrm{MA}^{(n)}$ [7, 8], for the single Holstein polaron model [9]: momentum-independent cou- pling between an optical phonon and a filled one-band system of non-interacting electrons. This is a highly oversimplified approach, in which strong interactions are not included as opposed to e.g. Ref.10, and even the effect of the Fermi sea is not accounted for (as there is no welldefined chemical potential, the latter will be positioned at the top of the first electron-removal state). Although not applicable to correlated electron systems, the plain Holstein polaron 9 is chosen as the minimalistic model to study the electron-phonon coupling problem. Since the MA ${ }^{(n)}$ has been shown to be extremely accurate everywhere in parameter space [8], it will allows us to study $A(\mathbf{k}, \omega)$ over a broad range of electron-phonon coupling.

Before delving into the detailed self-energy analysis, let us illustrate the model Hamiltonian and emphasize some general aspects, relevant to the phenomenological description of spectral functions in terms of effective coupling and renormalization parameters. Limiting ourselves to the one dimensional case for simplicity (higher dimensions were found not to change the results qualitatively), we have used the $\mathrm{MA}^{(n)}$ approximation to obtain self-consistent $A(k, \omega)$ with highly accurate momentumindependent $\Sigma(\omega)$, from the Holstein Hamiltonian:

$$
\mathcal{H}=\sum_{k} \varepsilon_{k}^{b} c_{k}^{\dagger} c_{k}+\Omega \sum_{Q} b_{Q}^{\dagger} b_{Q}+\frac{g}{\sqrt{N}} \sum_{k, Q} c_{k-Q}^{\dagger} c_{k}\left(b_{Q}^{\dagger}+b_{-Q}\right) .
$$

Its terms describe, in order, an electron with dispersion $\varepsilon_{k}^{b} \equiv-2 t \cos (k a)$, an optical phonon with energy $\Omega$ and momentum $Q$, and the on-site electron-phonon linear coupling [for $N$ sites with periodic boundary conditions; $c_{k}^{\dagger}\left(c_{k}\right)$ and $b_{Q}^{\dagger}\left(b_{Q}\right)$ are the usual electron and phonon creation (annihilation) operators]. This leads to a dimensionless effective coupling $\lambda \equiv g^{2} / 2 t \Omega$. For this paper we set $a=\hbar=1$ and $t=50 \mathrm{meV}$, such that the bandwidth is $200 \mathrm{meV}$ and the Brillouin zone is $2 \pi \AA^{-1}$ wide; also note that an additional constant $1 \mathrm{meV}$ FWHM Lorentzian broadening is applied, similar to an impurity scattering, to allow resolving the sharpest features in $A(k, \omega)$. 


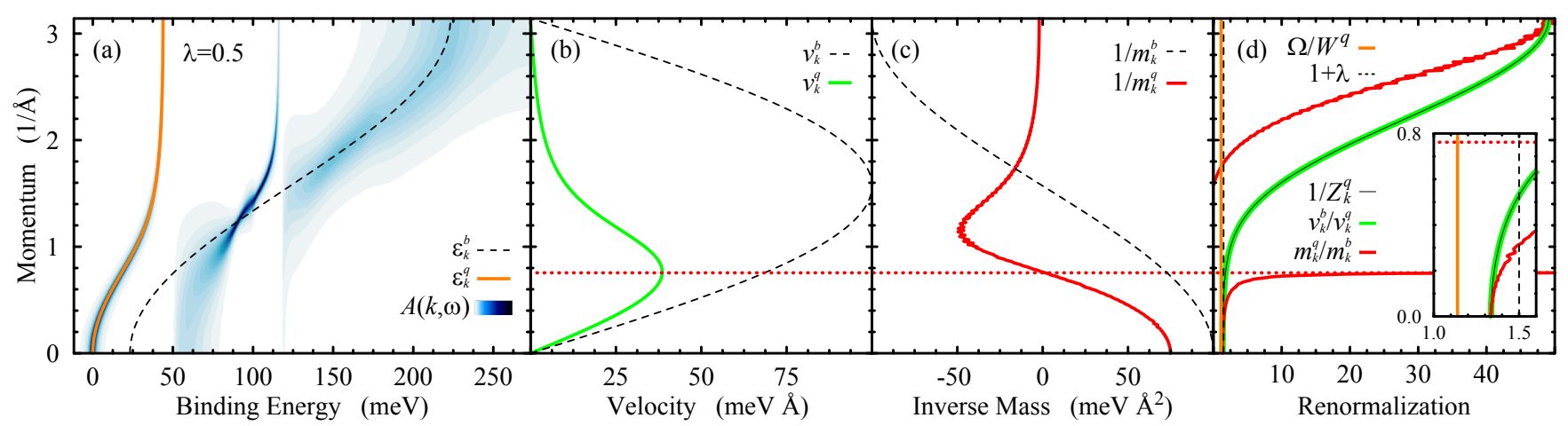

FIG. 1: (Color online). (a) $A(k, \omega)$ calculated within $\mathrm{MA}^{(1)}$ for $\Omega=50 \mathrm{meV}$ and $\lambda=0.5$; the quasiparticle dispersion $\varepsilon_{k}^{q}$ and the bare-band $\varepsilon_{k}^{b}$ are also shown. (b) Quasiparticle and bare-band velocities, $v_{k}^{q}$ and $v_{k}^{b}$, and (c) corresponding inverse masses, $1 / m_{k}^{q}$ and $1 / m_{k}^{b}$, according to the definitions $v_{k}=\partial \varepsilon_{k} / \partial k$ and $1 / m_{k}=\partial^{2} \varepsilon_{k} / \partial k^{2}$. (d) Momentum-dependent quasiparticle renormalization as obtained from $v_{k}^{b} / v_{k}^{q}, m_{k}^{q} / m_{k}^{b}$, and the inverse quasiparticle coherence $1 / Z_{k}^{q}$, where $Z_{k}^{q}=\int^{q} A(k, \omega) d \omega$ is the quasiparticle-only integrated spectral weight; in the inset, these quantities are compared near $k=0$ to the renormalization factors $\Omega / W^{q}$ and $(1+\lambda)$, obtained from quasiparticle bandwidth $W^{q}$ and dimensionless coupling $\lambda=g^{2} / 2 t \Omega$ in our model.

The spectral function calculated with $\mathrm{MA}^{(1)}$ for $\Omega=$ $50 \mathrm{meV}$ and $\lambda=0.5$ is presented as a false-color plot in Fig.1(a); as one can see, it deviates remarkably from the one of the uncoupled bare-band $\varepsilon_{k}^{b}$. The electronremoval spectrum is now comprised of a polaron quasiparticle band $\varepsilon_{k}^{q}$ (i.e., the lowest-energy bound-state of the electron-phonon coupled system), and a continuum of excitations starting at an energy $\Omega$ below the top of the polaron band and roughly following the original location of the bare-band $\varepsilon_{k}^{b}$. Based on these simulated data and on the precise knowledge of the input value of $\lambda$, it is possible to gauge the appropriateness of estimating the electron-phonon coupling strength from the observed renormalization of band velocities, masses, and quasiparticle coherence, as often done in the interpretation of ARPES results [1. To this end, in Fig. 1(b,c) we present the quasiparticle and bare-band velocities, $v_{k}^{q}$ and $v_{k}^{b}$, and the inverse masses, $1 / m_{k}^{q}$ and $1 / m_{k}^{b}$ (see caption of Fig. 1 for definitions). The corresponding ratios $v_{k}^{b} / v_{k}^{q}$ and $m_{k}^{q} / m_{k}^{b}$, as well as the inverse quasiparticle coherence $1 / Z_{k}^{q}$ (see again caption of Fig.1), are progressively larger than one the stronger the coupling strength; these quantities are usually equated to the renormalization or massenhancement factor $(1+\lambda)$, providing a potential path to the quantitative estimation of the electron-phonon coupling strength [11, 12].

As shown in Fig.1(d), the velocity, mass, and spectral weight quasiparticle renormalizations are functions of $k$. While in general one could expect these quantities to all be distinct, we find that $v_{k}^{b} / v_{k}^{q}=1 / Z_{k}^{q}$ at all $k$, which is a direct consequence of the $k$-independence of the self-energy derived from the Holstein Hamiltonian: by Taylor expanding $\Sigma(k, \omega)$ in the vicinity of the quasiparticle pole, i.e. $\omega=\varepsilon_{k}^{q}+\delta \omega$, one can approximate the Green's function as $G(k, \omega) \simeq Z_{k} /\left(\omega-\varepsilon_{k}^{q}\right)$ in terms of the quasiparticle coherence $Z_{k}^{q}=1 /\left[1-\partial \Sigma(k, \omega) /\left.\partial \omega\right|_{\omega=\varepsilon_{k}^{q}}\right]$, and obtain for the quasiparticle velocity $v_{k}^{q}=Z_{k}^{q}\left[v_{k}^{b}+\right.$ $\left.\partial \Sigma(k, \omega) /\left.\partial k\right|_{\omega=\varepsilon_{k}^{q}}\right]$; the latter reduces to $v_{k}^{q}=Z_{k}^{q} v_{k}^{b}$ if $\partial \Sigma / \partial k=0$. For the mass renormalization, we obtain $m_{k}^{q} / m_{k}^{b}=1 / Z_{k}^{q}$ only for $k=0$ and $\pi$, which is simply a consequence of the fact that at the band extrema the velocities are zero and the corresponding rate of change away from the extrema has to follow the acceleration [see Fig.1(d) and its inset; the horizontal dashed line emphasizes the divergence of $m_{k}^{q} / m_{k}^{b}$ due to the vanishing of $\left.1 / m_{k}^{q}\right]$. Most importantly, $v_{k}^{b} / v_{k}^{q}, m_{k}^{q} / m_{k}^{b}$, and $1 / Z_{k}^{q}$ cannot be compared directly to the momentum-independent renormalization factors $\Omega / W^{q}$ and $(1+\lambda)$, obtained from the quasiparticle bandwidth $W^{q}$ and the dimensionless

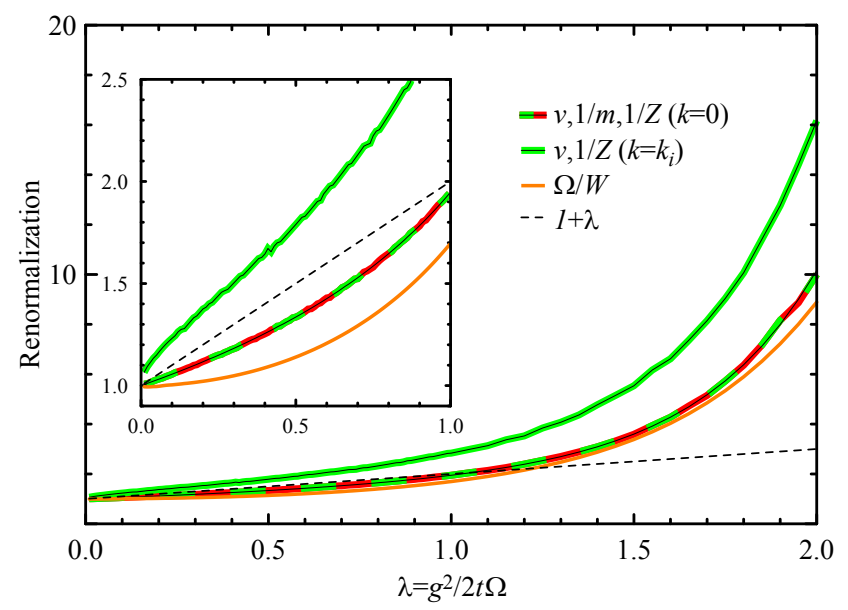

FIG. 2: (Color online). Renormalization parameters defined as in Fig. 1 plotted vs. the dimensionless coupling $\lambda=g^{2} / 2 t \Omega$. Note that the noise in $v$ and $1 / Z$ at $k=k_{i}$ originates from the determination of the inflection point $k_{i}$. 
coupling $\lambda=g^{2} / 2 t \Omega$ in our model [Fig.11(d), inset]. Although coincidences in values can be observed at some electron momenta, these are purely circumstantial and cannot be generalized.

The results in Fig. 1 demonstrate that in general one cannot directly extract a quantitative value for the coupling strength $\lambda$ from the observed renormalization of quasiparticle velocity, mass, and coherence (and this without even considering further complications originating from the electron-correlation driven renormalization of electron-phonon coupling in real materials [5, 13]). If one was to do that, the extracted value would vary with the chosen observable and the specific $k$ value, making any conclusion arbitrary (e.g., at $k=\pi$ these renormalizations overestimate $\lambda$ by a factor of almost 100). One may wonder, however, if any of these quantities scaled as $(1+\lambda)$ upon increasing $\lambda$, so that if not the exact value at least the trend of the coupling strength could be captured, for example in an experiment performed as a function of doping. In Fig.2 we follow each of these quantities and the quasiparticle bandwidth as a function of $\lambda$, contrasted against $(1+\lambda)$. For momentum dependent quantities we must choose a $k$ value: we plot $v_{0}^{b} / v_{0}^{q}, m_{0}^{q} / m_{0}^{b}$, and $1 / Z_{0}^{q}$ at $k=0$ where they all coincide, as well as $v_{k_{i}}^{b} / v_{k_{i}}^{q}$ and $1 / Z_{k_{i}}^{q}$ at the inflection point $k=k_{i}$ of the quasiparticle band $\varepsilon_{k}^{q}$ where $m_{k_{i}}^{q} / m_{k_{i}}^{b}$ diverges. All quantities deviate dramatically from $(1+\lambda)$ at large coupling values (e.g., overestimating the microscopic coupling by a factor of 4 to 8 at $\lambda=2$ ), and are also rather poor indicators of the coupling strength in the low-coupling regime $(\lambda<1$; Fig. 2 inset). Interestingly, the inflection point velocity renormalization $v_{k_{i}}^{b} / v_{k_{i}}^{q}$ is linear in $\lambda$ over a range that scales with the ratio $\Omega / t$. However, even this term deviates from $(1+\lambda)$ in a way dependent on the details of the model, such as the shape of the bare-band $\varepsilon_{k}^{b}$; in general, it cannot be used for the quantitative estimate of $\lambda$.

Determining $\varepsilon_{k}^{b}$ is a key step also in the attempt of extracting real and imaginary parts of the self-energy from $A(k, \omega)$, which will be the focus of the remainder of the paper. We analyze $A(k, \omega)$ in terms of MDCs at constant energy $\omega=\tilde{\omega}$. Since the self-energy in the present model is $k$-independent, i.e. $\Sigma_{\tilde{\omega}}^{\prime}$ and $\Sigma_{\tilde{\omega}}^{\prime \prime}$ are constant, as long as $\varepsilon_{k}^{b}$ can be linearized in the vicinity of the MDC peak maximum at $k=k_{m}$, the MDC lineshape is Lorentzian. Note that the converse is not true: a Lorentzian MDC lineshape is not a sufficient condition to conclude $\Sigma=\Sigma(\omega)$ [14, although the overlap of $v_{k}^{b} / v_{k}^{q}$ and $1 / Z_{k}^{q}$ (Fig.11) confirms the $k$-independence along the quasiparticle dispersion. By Taylor expanding $\varepsilon_{k}^{b}$ about the MDC peak maximum at $k=k_{m}$, i.e. $\varepsilon_{k}^{b}=\varepsilon_{k_{m}}^{b}+v_{k_{m}}^{b} \cdot\left(k-k_{m}\right)+\ldots$, and noticing that $\tilde{\omega}=\varepsilon_{k_{m}}^{b}+\Sigma_{\tilde{\omega}}^{\prime}$, we can rewrite Eq. 1 as:

$$
A_{\tilde{\omega}}(k) \simeq \frac{A_{0}}{\pi} \frac{\Delta k_{m}}{\left(k-k_{m}\right)^{2}+\left(\Delta k_{m}\right)^{2}},
$$

where $\Delta k_{m}=-\Sigma_{\tilde{\omega}}^{\prime \prime} / v_{k_{m}}^{b}$ is the half-width half-maximum

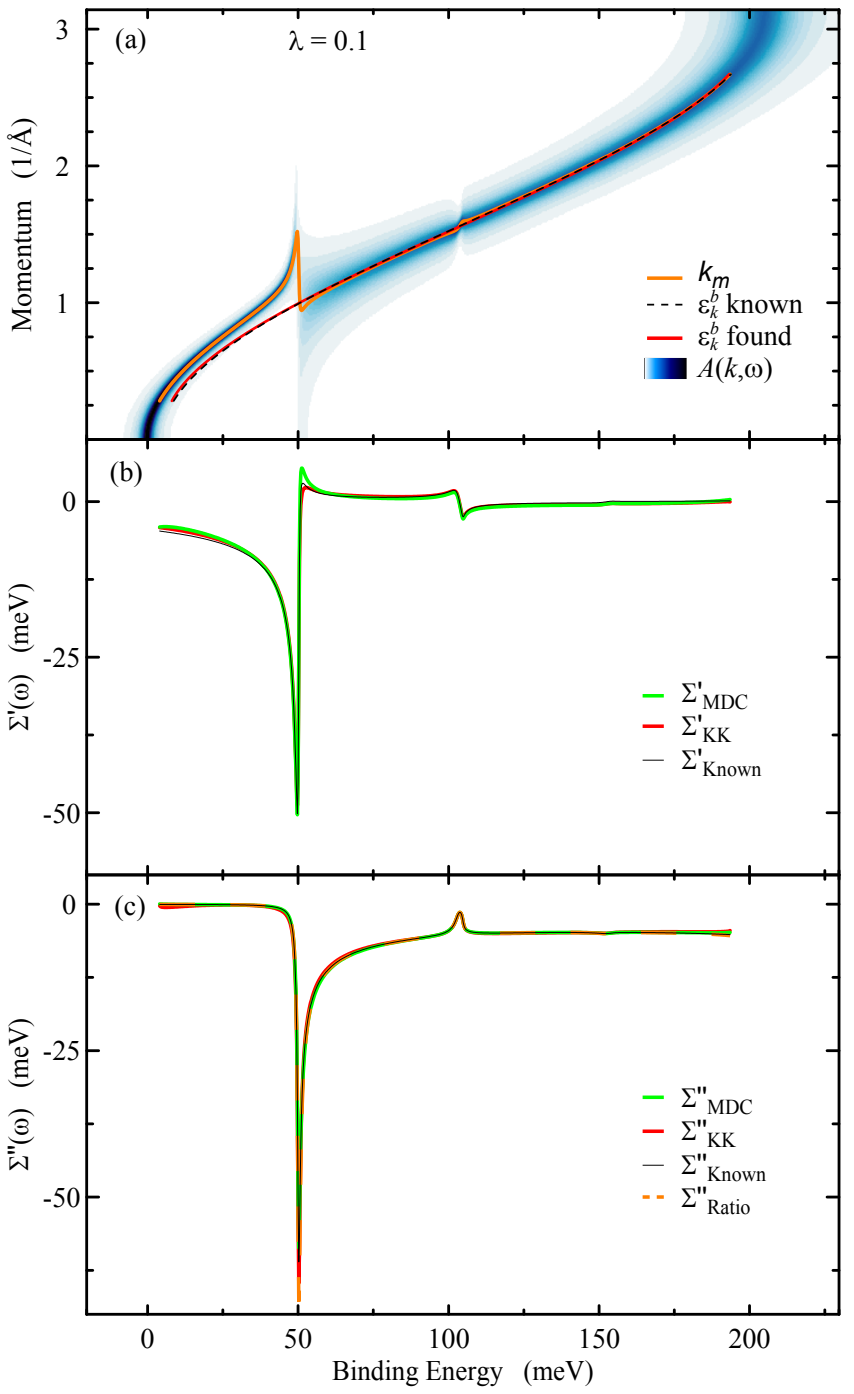

FIG. 3: (Color online). (a) $A(k, \omega)$ calculated within $\mathrm{MA}^{(1)}$ for $\Omega=50 \mathrm{meV}$ and $\lambda=0.1$; also shown are the $k_{m}$ path of MDC maxima, as well as the known bare-band and the one found through the KKBF analysis. (b,c) Real and imaginary part of the self-energy from the model $\left(\Sigma_{\text {known }}\right)$, the bareband and MDC fitting routine $\left(\Sigma_{M D C}\right)$, and the KK transform of $\Sigma_{M D C}^{\prime \prime}\left(\Sigma_{K K}^{\prime}\right)$ and $\Sigma_{M D C}^{\prime}\left(\Sigma_{K K}^{\prime \prime}\right)$. In (c) the MDC ratio results, $\Sigma_{\text {ratio }}^{\prime \prime}=-\Delta k_{m} / A_{0}$, are also shown.

of the Lorentzian MDC and $A_{0}=1 / v_{k_{m}}^{b}=\int A_{\tilde{\omega}}(k) d k$. If the bare-band is not known it is possible to fit it, within an arbitrary offset, to any functional form which provides a value and derivative using a Kramers-Kronig bare-band fitting (KKBF) routine. This is done by first tracking $k_{m}$ and $\Delta k_{m}$ for every $\tilde{\omega}$ through a Lorentzian fit of the MDC (Eq. 3), and then choosing $\varepsilon_{k}^{b}$ parameters such that $\Sigma_{M D C}^{\prime} \equiv \tilde{\omega}-\varepsilon_{k_{m}}^{b}$ and $\Sigma_{M D C}^{\prime \prime} \equiv-v_{k_{m}}^{b} \Delta k_{m}$ are self-consistent with $\Sigma_{K K}^{\prime}$ and $\Sigma_{K K}^{\prime \prime}$ calculated from the Kramers-Kronig (KK) relations:

$$
\Sigma_{K K}^{\prime, \prime \prime}(k, \omega)= \pm \frac{1}{\pi} \mathcal{P} \int_{-\infty}^{\infty} \partial \xi \frac{\Sigma_{M D C}^{\prime \prime}, \prime(k, \xi)}{\xi-\omega} .
$$



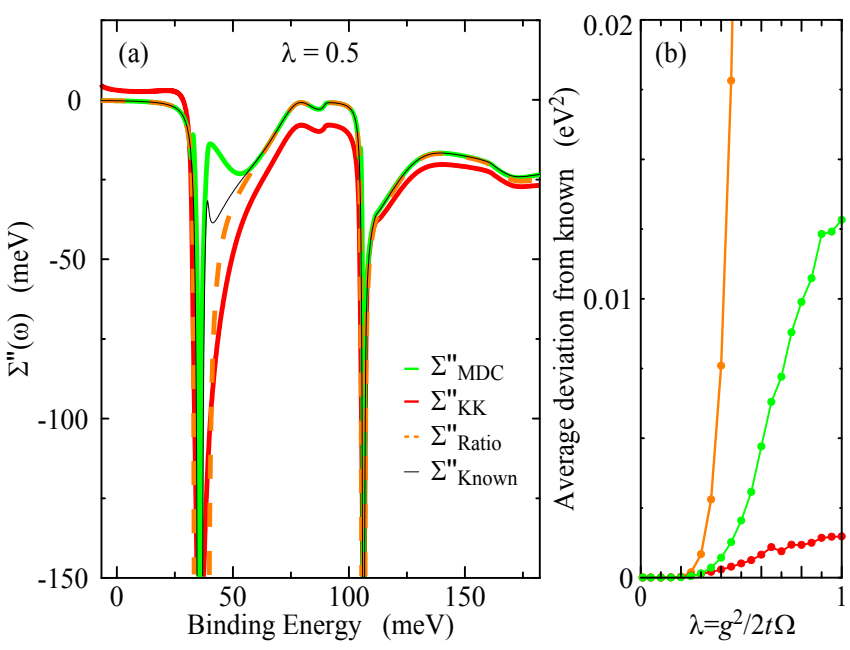

FIG. 4: (Color online). (a) Various estimates for the imaginary part of the self-energy, defined as in the caption of Fig. 3 for $A(k, \omega)$ calculated within $\mathrm{MA}^{(1)}$ for $\Omega=50 \mathrm{meV}$ and $\lambda=0.5$. (b) Deviation (i.e., average of the squared difference at each $k_{m}$ ) between estimated and known self-energies vs. $\lambda$.

In our implementation (Fig.3), a simple third order polynomial was used with an initial guess found by fitting MDC peak maxima. We then used the LevenbergMarquardt Algorithm as implemented in the mpfit package for IDL to vary band parameters. We found that the standard sum-of-squares minimization did not perform as well as a concave-down function, since it placed too much weight on outlying points far away. In order to evaluate the integrals in Eq.4 with a finite region of data, biased inverse polynomial fits where used to extrapolate tails before a Fourier-based transform was performed.

The method outlined here varies slightly from techniques previously described in the literature, which often deal with data very close to the Fermi energy and generally reduce the possible functional forms for $\varepsilon_{k}^{b}$ substantially [15, 16. While these methods present an exact solution for $A(k, \omega)$ based on a reduced bare-band, the present approach imposes no restrictions on $\varepsilon_{k}^{b}$ (other than it be differentiable near $k_{m}$ ), allowing fitting based on a wider variety of bare-band models and using KKBF to vary $\varepsilon_{k}^{b}$ parameters. The results of the KKBF procedure are presented for $\lambda=0.1$ in Fig. 3 and show that, in the low-coupling regime, the found bare-band and selfenergies $\left(\Sigma_{M D C}\right)$ agree well with the known quantities from our model (as long as the MDC analysis is applicable: $k_{m}$ is far from band extrema where velocities vanish, and MDC widths are suitably small that the bare-band may be approximated as linear around $k_{m}$ ). Note that the self-energies are evaluated along the $\left(k_{m}, \tilde{\omega}\right)$-path of the MDC maxima, along which the analysis is performed; this path deviates significantly from either $\varepsilon_{k}^{q}$ or $\varepsilon_{k}^{b}$ close to the sharper one- and two-phonon structures [Fig. 3(a)]. The reliability of the results is confirmed by the agree- ment between $\Sigma_{M D C}$ and $\Sigma_{K K}$ over the whole range, which provides an internal self-consistency check.

Although very satisfactory at low coupling, at larger coupling the results from the KKBF procedure become progressively less accurate. This can be seen already for $\lambda=0.5$ in Fig.4 $4\left(\right.$ a), where $\Sigma_{M D C}^{\prime \prime}$ and $\Sigma_{K K}^{\prime \prime}$ fail to reproduce $\Sigma_{\text {known }}^{\prime \prime}$ in the proximity of the sharp phononinduced structures $\left(\Sigma_{K K}^{\prime \prime}\right.$ has also picked up different offsets in the different flatter parts of the spectrum, making setting its overall offset difficult). For a more comprehensive description of the deviation of these quantities from $\Sigma_{\text {known }}^{\prime \prime}$ upon increasing $\lambda$, in Fig. $4(\mathrm{~b}$ (b) we present the average of the squared difference at each $k_{m}$ between the estimated and known self-energies versus $\lambda$ : for both $\Sigma_{M D C}^{\prime \prime}$ and $\Sigma_{K K}^{\prime \prime}$, this estimate shows a rapid and consistent increase with $\lambda$, indicating a failure of the KKBF analysis and similar methods in the intermediate to strong electron-phonon coupling regime.

Before concluding, we will point out an alternative, possibly more practical approach, which allows us to tackle the problem over a larger range of $\lambda$, at least for $\Sigma^{\prime \prime}$. In our method, determining real and imaginary parts of the self-energy hinges on finding a proper expression for the bare-band through the KKBF routine. The imaginary part however, $\Sigma_{\tilde{\omega}}^{\prime \prime}=-v_{k_{m}}^{b} \Delta k_{m}$, only requires knowledge of $v_{k_{m}}^{b}$, and this can be obtained directly from $A(k, \omega)$ in two independent ways. The first one is through the momentum integral of $A_{\tilde{\omega}}(k)$ in Eq. 3 , which returns directly $A_{0}=1 / v_{k_{m}}^{b}$; this allows a simple estimate of $\Sigma^{\prime \prime}$ [and equally accurate to KKBF's for $\lambda=0.1$, Fig.3. (c)] from the MDC width/integral ratio: $\Sigma_{\text {ratio }}^{\prime \prime}=-\Delta k_{m} / A_{0}$. The second one is through the equivalence $v_{k}^{b} / v_{k}^{q}=1 / Z_{k}^{q}$ discussed in the context of Fig.11(d), and the possibility of estimating $v_{k}^{q}$ and $Z_{k}^{q}$ directly from the data as the momentum derivative and energy integral of the quasiparticle band $\varepsilon_{k}^{q}$ (Fig. 1): $\Sigma_{M D C}^{\prime \prime}=-v_{k}^{q} \Delta k_{m} / Z_{k}^{q}$. The so-obtained $\Sigma_{\text {ratio }}^{\prime \prime}$ and $\Sigma_{M D C}^{\prime \prime}$ are compared in Fig. 4(a); although especially $\Sigma_{\text {ratio }}^{\prime \prime}$ deviates strongly from $\Sigma_{\text {known }}^{\prime \prime}$ upon increasing $\lambda$ at the sharp phonon-induced features [Fig. $4(\mathrm{~b})]$, the general behavior is that when $\Sigma_{\text {ratio }}^{\prime \prime} \approx$ $\Sigma_{M D C}^{\prime \prime}$ they also match $\Sigma_{\text {known }}^{\prime \prime}$ almost exactly. Thus, $\Sigma_{\text {ratio }}^{\prime \prime}$ and $\Sigma_{M D C}^{\prime \prime}$ can be used to obtain a precise anchor mesh for $\Sigma^{\prime \prime}$ over a range of energies, without the critical step of finding the bare-band through KKBF.

In summary we have shown that, at variance with a common phenomenological practice in the interpretation of ARPES data, even in the simplest case of electronphonon coupling described by the Holstein model it is not possible to obtain the microscopic coupling strength from the observed renormalization of quasiparticle coherence, velocity, mass, or bandwidth (e.g., $v_{k_{i}}^{b} / v_{k_{i}}^{q}$ would overestimate $\lambda$ by a factor of 2,3 , and 8 for $\lambda=1,1.5$, and $2)$. In this sense, the coupling $\lambda$ still remains an elusive quantity. Through the KKBF analysis we can however gain access to bare-band and self-energies, which if properly modelled might provide information on the nature 
and strength of the underlying interactions, at least in the momentum independent, low-coupling regime.

We gratefully acknowledge S. Johnston, T.P. Devereaux, F. Marsiglio, and G.A. Sawatzky for many useful discussions. This work was supported by the Killam Program (A.D.), the Alfred P. Sloan Foundation (M.B. and A.D.), CRC Program (A.D.), NSERC, CFI, CIFAR Quantum Materials and Nanoelectronics Programs, and BCSI.

* Electronic address: damascelli@physics.ubc.ca

[1] A. Damascelli, Physica Scripta T109, 61 (2004).

[2] T. Valla et al., Phys. Rev. Lett. 83, 2085 (1999).

[3] Z.-X. Shen et al., Phil. Mag. B 82, 1349 (2002).

[4] T. K. Kim et al., Phys. Rev. Lett. 91, 167002 (2003).
[5] N. Ingle et al., Phys. Rev. B 72, 205114 (2005).

[6] A. Bostwick et al., Science 328, 999 (2010).

[7] M. Berciu, Phys. Rev. Lett. 97, 036402 (2006).

[8] M. Berciu and G. L. Goodvin, Phys. Rev. B 76, 165109 (2007).

[9] T. Holstein, Ann. Phys. 8, 325 (1959); ibid., 343 (1959).

[10] P. Werner and A. Millis, Phys. Rev. Lett. 99, 146404 (2007).

[11] Ashcroft and Mermin, Solid State Physics (Thomson Learning, 1976).

[12] E. G. Maksimov, M. L. Kulic, and O. V. Dolgov, arXiv:1001.4859 (2010).

[13] P. Benedetti and R. Zeyher, Phys. Rev. B 59, 9923 (1999).

[14] M. Randeria, A. Paramekanti, and N. Trivedi, Phys. Rev. B 69, 144509 (2004).

[15] A. A. Kordyuk et al., Phys. Rev. B 71, 214513 (2005).

[16] A. Kaminski and H. M. Fretwell, New J. Phys. 7, 98 (2005). 